\title{
A Flexible Polynomial Expansion Method for Response Analysis with Random Parameters
}

\author{
Rugao Gao $\mathbb{D}^{1,2}$ Keping Zhou, ${ }^{1,2}$ and Yun Lin $\mathbb{D}^{1,2}$ \\ ${ }^{1}$ Central south university, School of resources and safety engineering, Hunan Changsha, Hunan, 410083, China \\ ${ }^{2}$ Hunan Key Laboratory of Mineral Resources Exploitation and Hazard Control for Deep Metal Mines, Changsha 410083, China
}

Correspondence should be addressed to Yun Lin; yunlin617@csu.edu.cn

Received 20 July 2018; Revised 11 October 2018; Accepted 11 November 2018; Published 3 December 2018

Academic Editor: Michele Scarpiniti

Copyright (C) 2018 Rugao Gao et al. This is an open access article distributed under the Creative Commons Attribution License, which permits unrestricted use, distribution, and reproduction in any medium, provided the original work is properly cited.

\begin{abstract}
The generalized Polynomial Chaos Expansion Method (gPCEM), which is a random uncertainty analysis method by employing the orthogonal polynomial bases from the Askey scheme to represent the random space, has been widely used in engineering applications due to its good performance in both computational efficiency and accuracy. But in gPCEM, a nonlinear transformation of random variables should always be used to adapt the generalized Polynomial Chaos theory for the analysis of random problems with complicated probability distributions, which may introduce nonlinearity in the procedure of random uncertainty propagation as well as leading to approximation errors on the probability distribution function (PDF) of random variables. This paper aims to develop a flexible polynomial expansion method for response analysis of the finite element system with bounded random variables following arbitrary probability distributions. Based on the large family of Jacobi polynomials, an Improved Jacobi Chaos Expansion Method (IJCEM) is proposed. In IJCEM, the response of random system is approximated by the Jacobi expansion with the Jacobi polynomial basis whose weight function is the closest to the probability density distribution (PDF) of the random variable. Subsequently, the moments of the response can be efficiently calculated though the Jacobi expansion. As the IJCEM avoids the necessity that the PDF should be represented in terms of the weight function of polynomial basis by using the variant transformation, neither the nonlinearity nor the errors on random models will be introduced in IJCEM. Numerical examples on two random problems show that compared with gPCEM, the IJCEM can achieve better efficiency and accuracy for random problems with complex probability distributions.
\end{abstract}

\section{Introduction}

Nowadays, design and optimization of engineering systems heavily rely on numerical methods in industry. This is especially true in the acoustic field, where both time and economic costs of experiment are particularly expensive. In the past decade, the uncertainty analysis methods in the prediction of acoustic field has been greatly developed [15]. Generally, uncertainty analysis methods can be classified as two distinct groups: probabilistic methods $[6,7]$ and nonprobabilistic methods [8-12]. The probabilistic method is the optimal choice for uncertainty analysis once the PDF is derived [13].

The Monte Carlo method (MCM) is a simple and robust probabilistic method, and thus it has been widely used for random analysis [14]. However, the convergence rate of MCM is rather slow and the corresponding computational cost of MCM for the uncertainty analysis of massive engineering projects is tremendous. In order to improve the efficiency of MCM, some advanced MCMs have been developed, such as the Latin hypercube sampling simulation method [15], the quasi Monte Carlo method [16], and the other extended methods [17-19]. The convergence rate of advanced MCMs will become faster than the ordinary MCM. However, additional restrictions are posed on the design of these advanced MCMs and their applicability is often limited [20]. Generally, in order to verify the accuracy of uncertain methods, ordinary MCM is still considered as the most robust sampling method.

The perturbation stochastic method is also a key category of probabilistic methods, where the response of random systems is expanded as the Taylor series around the mean 
value of random variables and truncated at a certain order [21-23]. Typically, the first-order expansion is employed in Taylor series, as application of high-order Taylor expansion does not allow for significant improvements in accuracy. Due to the high computational efficiency, the perturbation stochastic method has been used in various engineering fields [24-26]. An inherent limitation of perturbation stochastic methods is that it is limited to random problem with small uncertainty level.

During the past decades, the generalized Polynomial Chaos Expansion Method (gPCEM) has gained a large popularity in the field of random uncertainty analysis [27]. The development of gPCEM started with the work of R. Ghanem et al., who used Hermite polynomial as the basis for the analysis of stochastic processes [28, 29]. Subsequently, the gPCEM which is an extension of Hermite expansion method, was derived based on the Askey Scheme [3032]. Within gPCEM, different kinds of orthogonal polynomials can be chosen as basis to establish the surrogate model for the random response. Thus, the gPCEM can achieve higher accuracy for some random problems than the Hermite polynomial expansion, such as the random problems with Beta distribution and uniform distribution. The variance and standard deviation of gPCEM can be obtained efficiently when compared to the conventional MCM. In addition, the law of approximation of generalized polynomial chaos expansion is rather mature [33-35]. Due to the advantages mentioned above, the gPCEM is very important probabilistic method for solving random problems [36-39].

Generally speaking, the gPCEM has been widely popularized in random problem analysis. However, it seems the gPCEM can only be directly employed to expand the response of random systems with the so called basic random variable, whose PDF can be orthogonal with respect to the polynomial in Askey Scheme. For the random problems with nonbasic random variable, a non-linear transformation should be employed, through which the non-basic random variable can be approximately represented as a nonlinear function of basic random variables. Nevertheless, two inherent issues will be encountered in the transformation of nonbasic random variables. First, the non-linearity will be introduced by using the nonlinear transformation, which may lead to slow convergence of the expansion. Second, even if the high-order nonlinear transformation is used to approximate the real PDF in terms of the weight function, some fitting errors between the approximated PDF and the real PDF may be introduced, especially when the real PDF of random variable is not a smooth function. If there is large fitting error, the moment of response obtained by gPCEM may be failed to converge to the exact result. To decrease the fitting error by using the nonlinear transformation, Multielement gPCEM is proposed by Xiu et al. The Multielement gPCEM can be applied for random problems with arbitrary probability distributions by discretizing the random space into piecewise random elements and construct the polynomial expansion locally in each random element [39-43]. However, the discretizing of probability distributions can lead to tremendous computational effort for the random problems with multiple input variables [44]. As regarding the solution of engineering problems, the uncertain problems always involve complex probability distribution as well as large number of variables. Thus, it is desirable to improve the application value of generalized polynomial theory in stochastic problems, with fine ruggedness and strong adaptability.

Moreover, though the gPCEM has received much attention lately, few engineering problems are analyzed with generalized polynomial chaos theory [45]. The analysis of the effects of vibration and noise is one of the key points for the design of engineering systems with high level NVH performance. During the last decades, lots of uncertainty analysis methods based on the perturbation theory have been proposed for random response analysis of acoustic problem [46-50]. However, the perturbation based method can only solve random problems with small uncertainty level [50]. Recently, in the interval and random analysis of acoustic system, the Gegenbauer series was introduced to establish the surrogate model [45]. The accuracy of Gegenbauer series expansion is significantly improved. Nevertheless, the Gegenbauer series expansion method is only suitable for simple uncertain problems, which may limit its applications in the real acoustic problems.

The main purpose of this paper is to develop a powerful polynomial expansion method for the response analysis of acoustic systems with random variables following arbitrary probability distribution. As the uncertain parameter of acoustic systems is always bounded in real engineering, the Jacobi polynomial that is defined in a bounded interval will be introduced. Based on the Jacobi polynomial, an Improved Jacobi Chaos Expansion Method (IJCEM) is proposed in this paper. In IJCEM, the response of acoustic systems in the range of variation of random variables is approximated by the Jacobi expansion. A polynomial selection criterion is established to find the best polynomial basis from the large family of Jacobi polynomials for the approximation of response of acoustic systems with different probability distributions of random variables. The mean and variance of response are calculated through the Jacobi expansion. For the random variable with Beta distribution, the mean and variance of Jacobi expansion can be obtained analytically and expressed by the coefficients of Jacobi expansion. For the random variable with non-Beta distribution, the mean and variance of Jacobi expansion are obtained by using the Monte Carlo simulation. Note that the Jacobi expansion is a simple function, thus the computational burden suffering from the Monte Carlo simulation of Jacobi expansion is acceptable. The proposed method was employed to predict the response of acoustic systems with random variables following complex probability distributions. Numerical results have shown that the proposed IJCEM can achieve higher accuracy and efficiency than that of the gPCEM.

The main contents of this paper are as follows: Section 2 presents a stochastic model of acoustic response. Section 3 briefly summarizes the features and deficiencies of gPCEM analysis. In Section 4, the operation of ITJEM is illustrated. The characteristics and feasibility of this method are described in Section 5. Section 6 summarizes the research work in this paper. 


\section{Random Model of Uncertain Acoustic Problems}

The equilibrium equation affected by harmonics can be expressed as

$$
\left(\mathbf{K}-k^{2} \mathbf{M}+\mathrm{j} k \mathbf{C}\right) \mathbf{p}=\mathbf{F}
$$

where $\mathrm{j}=\sqrt{-1}$ is an imaginary unitary, $k=\omega / c$ is the wave number, $\omega$ is the angular frequency, $c$ is the acoustic speed of fluid, and $\mathbf{K}$ is the acoustic system stiffness matrix; $\mathbf{M}$ is the acoustic system mass matrix; $\mathbf{C}$ is the acoustic system damping matrix; $\mathbf{p}$ stands for the sound pressure vector; $\mathbf{F}$ stands for the acoustic system load vector. Equation (1) can be queried in [47].

There are many inherent uncertainties in the engineering sound field, such as uncertain physical property of acoustic medium caused by the variation of temperature and the uncertain velocity exciting produced by unpredictable environment. In most cases, these uncertainties can be modeled by random variables. Besides, the uncertain parameters of acoustic systems are always bounded in practice and the uncertain parameters of acoustic field can be described as bounded random variables. Using the bounded random variable vector $\mathbf{x}=\left[x_{1}, x_{2}, \ldots, x_{L}\right],(1)$ can be rewritten as

$$
\mathbf{Z}(\mathbf{x}) \mathbf{p}(\mathbf{x})=\mathbf{F}(\mathbf{x})
$$

where $\mathbf{F}(\mathbf{x})$ is the random force vector and $\mathbf{Z}(\mathbf{x})$ is the uncertain dynamic stiffness matrix, which can be expressed as follows:

$$
\mathbf{Z}(\mathbf{x})=\mathbf{K}(\mathbf{x})-k(\mathbf{x})^{2} \mathbf{M}(\mathbf{x})+\mathrm{j} k(\mathbf{x}) \mathbf{C}(\mathbf{x})
$$

where $k(\mathbf{x})$ stands for the random wave number and $\mathbf{K}(\mathbf{x})$, $\mathbf{M}(\mathbf{x})$, and $\mathbf{C}(\mathbf{x})$ stand for the random stiffness, mass, and damping matrix, respectively.

For convenience, we can transform the random variable $x_{i}(i=1,2, \ldots, L)$ into an unitary bounded random variable $\xi_{i}(i=1,2, \ldots, L)$ that is defined in $[-1,1]$. The linear transformation for a random vector $\mathbf{x}$ can be expressed as

$$
\begin{aligned}
& \mathbf{x}=\mathbf{x}(\xi), \quad \xi=\left[\xi_{1}, \xi_{2}, \ldots, \xi_{L}\right] \\
& x_{i}=x_{i}\left(\xi_{i}\right)=x_{i, 0}+x_{i, 1} \xi_{i}, \quad i=1,2, \ldots, L
\end{aligned}
$$

where

$$
\begin{aligned}
& x_{i, 0}=\frac{\bar{x}_{i}+\underline{x}_{i}}{2}, \\
& x_{i, 1}=\frac{\bar{x}_{i}-\underline{x}_{i}}{2}
\end{aligned}
$$

$\bar{x}_{i}$ and $\underline{x}_{i}$ stand for the upper and lower bounds of $x_{i}$, respectively. As a result, the response of the random model of acoustic systems can be expressed as

$$
\mathbf{p}(\boldsymbol{\xi})=\mathbf{Z}^{-1}(\mathbf{x}(\xi)) \mathbf{F}(\mathbf{x}(\boldsymbol{\xi}))
$$

\section{3. gPCEM for Random Variables}

In gPCEM, the Askey polynomial is used as the basis for interpretation. Xiu et al. proposed that any second-order process of L2 sense can be realized with such methods. [20]. In order to analytically calculate the moment of response, the PDF in gPCEM is expressed by the polynomial basis weight function. For convenience, the probability distribution whose $\mathrm{PDF}$ is the same as one of the weight function of polynomial basis in the Askey scheme is named as the basic probability distribution in this paper.

3.1. gPCEM for Random Uncertainty Analysis with Basic Probability Distribution. For the random problems with basic probability distribution, the response of random system can be expanded as

$$
Y(\xi)=\sum_{i=0}^{\infty} c_{i} \phi_{i}(\xi)
$$

where $c_{i}$ represents the expansion coefficients to be estimated and $\phi_{i}(\xi)$ denotes the generalized polynomial basis belonging to the Askey scheme. The type of $\phi_{i}(\xi)$ varies for different random problems. For random variables defined in $[-\infty,+\infty]$, the bases are the generalized Hermite polynomials; for random variables defined in a semibounded domain $[0,+\infty]$, the bases are the generalized Laguerre polynomials; for random variables defined in a bounded interval $[-1,1]$, the bases are the Jacobi polynomials; and more bases for other types of random variables can be found in [27].

Random variables of sound are usually constrained. The one-dimensional Jacobi polynomials can be given as

$$
\begin{aligned}
& J_{0}^{\alpha, \beta}(\xi)=1 \\
& J_{1}^{\alpha, \beta}(\xi)=\frac{1}{2}(\alpha+\beta+2) \xi+\frac{1}{2}(\alpha-\beta), \\
& J_{n}^{\alpha, \beta}(\xi)=\frac{J_{n-1}^{\alpha, \beta}(\xi) B_{0}+J_{n-2}^{\alpha, \beta}(\xi) C_{0}}{A_{0}}, \quad n=2,3, \ldots
\end{aligned}
$$

where

$$
\begin{aligned}
A_{0} & =2 n(n+\alpha+\beta)(2 n+\alpha+\beta-2) \\
B_{0} & =(2 n+\alpha+\beta-1) \\
& \cdot\left\{(2 n+\alpha+\beta)(2 n+\alpha+\beta-2) \xi+\alpha^{2}-\beta^{2}\right\} \\
C_{0} & =-2(n+\alpha-1)(n+\beta-1)(2 n+\alpha+\beta)
\end{aligned}
$$

$\alpha$ and $\beta$ are the Jacobi parameters that satisfied $\alpha>$ $-1, \beta>-1$. For L-dimensional random variables, the Jacobi polynomials can be expressed as the tensor product of the one-dimension Jacobi polynomial, which can be given by

$$
J_{k_{1}, \ldots, k_{L}}^{\alpha, \beta}(\boldsymbol{\xi})=\prod_{i=1}^{L} J_{k_{i}}^{\alpha, \beta}\left(\xi_{i}\right), \quad k_{i}=0,1,2, \ldots
$$


The Jacobi polynomials are orthogonal with respect to $w^{\alpha, \beta}(\xi)$, which can be expressed as

$$
\left\langle J_{i}^{\alpha, \beta}(\xi), J_{k}^{\alpha, \beta}(\xi)\right\rangle_{w}= \begin{cases}h_{k}, & k=i \\ 0, & k \neq i\end{cases}
$$

where

$h_{k}$

$$
=\frac{\Gamma(k+\alpha+1) \Gamma(k+\beta+1) \Gamma(\alpha+\beta+1)}{(2 k+\alpha+\beta+1) \Gamma(k+1) \Gamma(k+\alpha+\beta+1) \Gamma(\alpha+1) \Gamma(\beta+1)}
$$

$\langle\cdot, \cdot\rangle$ denotes the ensemble average which is the inner product in the Hilbert space of the random variable $\xi$, which can be expressed as

$$
\begin{aligned}
& \left\langle J_{i}^{\alpha, \beta}(\xi), J_{k}^{\alpha, \beta}(\xi)\right\rangle_{w} \\
& \quad=\int_{-1}^{1} w^{(\alpha, \beta)}(\xi) J_{i}^{(\alpha, \beta)}(\xi) J_{k}^{(\alpha, \beta)}(\xi) \mathrm{d} \xi
\end{aligned}
$$

$w^{\alpha, \beta}(\xi)$ is the weight function related to the Jacobi polynomial, which is given by

$$
\begin{aligned}
& w^{(\alpha, \beta)}(\xi) \\
& \quad=\frac{\Gamma(\alpha+\beta+1)}{2^{\alpha+\beta+1} \Gamma(\alpha+1) \Gamma(\beta+1)}(1+\xi)^{\alpha}(1-\xi)^{\beta}
\end{aligned}
$$

Based on the Jacobi polynomial, the response of interest for a one-dimension problem can be rewritten as the Jacobi expansion

$$
Y(\xi)=\sum_{i=0}^{\infty} c_{i} J_{i}^{\alpha, \beta}(\xi)
$$

Based on the theory of the Jacobi expansion, the coefficient $c_{i}$ can be derived as

$$
\begin{aligned}
c_{i} & =\frac{\left\langle Y(\xi), J_{i}^{\alpha, \beta}(\xi)\right\rangle}{\left\langle\left(J_{i}^{\alpha, \beta}\right)^{2}(\xi)\right\rangle} \\
& =\frac{1}{\left\langle\left(J_{i}^{\alpha, \beta}(\xi)\right)^{2}\right\rangle} \int Y(\xi) J_{i}^{\alpha, \beta}(\xi) w^{\alpha, \beta}(\xi) \mathrm{d} \xi
\end{aligned}
$$

Since the weight function is equal to the PDF of random variables, the mean and variance of $\mathrm{Y}$ can be derived from orthogonality analysis as follows [30]:

$$
\begin{aligned}
\mu(Y) & =c_{0} \\
\sigma^{2}(Y) & =\sum_{i=0}^{\infty}\left(c_{i}\right)^{2} h_{i}-\left(c_{0}\right)^{2}
\end{aligned}
$$

3.2. gPCEM for Random Uncertainty Analysis with Nonbasic Probability Distribution. In a wide range of engineering applications, the basic probability distribution is insufficient to describe the random process. To deal with other bounded probability distributions, a nonlinear variable transformation is employed such that a complex probability distribution can be represented by the weight function of polynomial basis. For instance, the nonlinear transformation for an arbitrary random variable $\mathrm{x}$ can be written as

$$
x \approx \tilde{x}=b_{0}+b_{1} \xi+b_{2} \xi^{2}+\cdots
$$

where $\xi$ is the random variables with basic probability distribution and $b_{0}, b_{1}, b_{2}, \ldots$ are the constant coefficients of nonlinear transformation, which can be given by

$$
\begin{aligned}
& \text { To find } b_{k}(k=0,1,2, \ldots) \\
& \min \int_{-1}^{1}\left\{P_{X}(x)-P_{\widetilde{X}}(x)\right\} \mathrm{d} x
\end{aligned}
$$

In the above equation, $P_{X}(x)$ is the original PDF of $x$ and $P_{\widetilde{X}}(x)$ is the PDF of function $\tilde{x}$.

By using the variable transformation, the gPCEM can also be extended to calculate the uncertain questions with nonbasic variables. Nevertheless, the computational efficiency of gPCEM will be seriously deteriorated when the variable is transformed as a high-order polynomial function of the basic random variable. The main reason is that the variable transformation can introduce the nonlinearity in the process of uncertainty propagation. Besides, the variable transformations may lead to some fitting errors between the approximated PDF and the original PDF, especially when the $\mathrm{PDF}$ of random variable is not a smooth function. If there is large fitting error, the response average value and variance derived by gPCEM may failed to converge to the exact result.

\section{IJCEM for Random Uncertainty Analysis of Sound with Arbitrary Probability Distributions}

To avoid the variable transformation when using generalized Polynomial Chaos theory to solve random problems with complicated probability distributions, an Improved Jacobi Chaos expansion Method (IJCEM) is developed in this section. In the developed method, the sound response is directly expanded in the application of the Jacobi polynomials. The core of the method is to realize the inversion of random problems in the acoustic system by polynomial expansion.

4.1. Determine Polynomial Parameters of Jacobi Expansion. Theoretically, all Jacobian polynomials defined on $[-1,1]$ can be used for expansion and then invert the uncertain response of the acoustic system, but the accuracy of the Jacobi method with different parameters of the corresponding polynomial bases may vary greatly as the weighted projection is a way for controlling the residual deviation of Jacobi method [30]. Reference [24] has shown that if the type of polynomial is used to match the import variable distribution, the fastest 


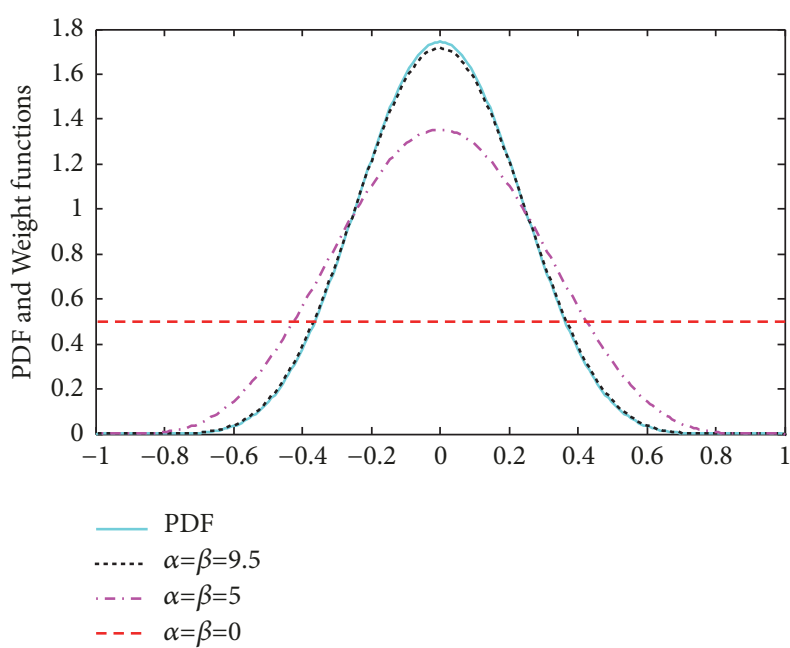

FIGURE 1: PDF of a random variable and the weight function with different polynomial parameters.

convergence rate will be implemented. In other words, the optimal polynomial basis can be determined by the same amount as the PDF for random problem analysis. However, in most engineering cases, the variable weighting value that equals the PDF of uncertain variable cannot be found from the Askey sckeme. As a result, the weighting function of polynomials basis of the Jacobi method to solve a random problem may be always deviated from the PDF of real uncertain variables if the variable transformation is not employed. In this paper, the deviation between the weight function and the PDF is allowed and a polynomial selection criterion will be established to choose the basis of Jacobi expansion. Before establishing a polynomial selection criterion, the influence of the residual between the variable weighting value and the PDF on the expansion will be discussed. The degree of residual between the weighting dependent variable and the $\mathrm{PDF}$ of a random variable can be define by a proximity index as follows:

$$
\delta=\delta(\alpha, \beta)=\lg \left(\int_{\Omega}\left|\frac{P_{X}(x)-w^{\alpha, \beta}(x)}{w^{\alpha, \beta}(x)}\right| \mathrm{d} x\right)
$$

$P_{X}(x)$ denotes the PDF of $\mathrm{x} ; w^{(\alpha, \beta)}(x)$ is the weight function related to the Jacobi polynomial with the Jacobi parameters $\alpha$ and $\beta$; $\delta$ denotes the proximity index of $w^{(\alpha, \beta)}(x)$ for $P_{X}(x)$.

A simple mathematic example will be considered to investigate the effect of $\delta$ on the convergence behavior of Jacobi expansion for random uncertainty qualification.

Example. Consider a function $y=\tan x$, where $\mathrm{x}$ is a uncertainty limited in $[-1,1]$. The probability distribution function of $x$ is shown Figure 1. The Jacobi expansion with different polynomial parameters are used to approximate the function $y$, and then the mean and variance of $y$ are calculated through MCM of y. Three cases when the Jacobi parameters are $\alpha=\beta=0, \alpha=\beta=5$ and $\alpha=\beta=9.5$, will be considered. For comparison, the weight function of Jacobi
TABLE 1: Proximity index of the weight functions with different Jacobi parameters for a PDF.

\begin{tabular}{lccc}
\hline Polynomial parameters & $\alpha=\beta=9.5$ & $\alpha=\beta=5.5$ & $\alpha=\beta=0$ \\
\hline Proximity index $\delta$ & 0.9 & 4.6 & 9.7 \\
\hline
\end{tabular}

polynomials related to each group of Jacobi parameters is also plotted in Figure 1. According to Eq. (21), the proximity index of the weight functions with different Jacobi parameters for the PDF shown in Figure 1 can be obtained, which are listed in Table 1. The Jacobi expansions with different Jacobi parameters are used to derive the mean and square deviation of $y$. The convergence behavior of the expansion is shown in Figure 2.

From Figure 2, we can find that the convergence speed of Jacobi expansion is improved with the decrease of $\delta$. The error of the mean and variance of y calculated by using Jacobi expansion with any $\delta$ can eventually decrease to le03 when the retained order is up to 7. Therefore, we can conclude from Figure 2 that (1) the Jacobi expansion with any Jacobi parameters can achieve high accuracy if the reserved sequences are large enough; (2) the converge speed of Jacobi expansion method can be improved by reducing $\delta$.

Based on the above conclusions, we can define the polynomial selection criterion by an optimization solution as follows:

$$
\begin{aligned}
\min & \delta(\alpha, \beta) \\
\text { s.t. } & \alpha, \beta>-1
\end{aligned}
$$

Through the above optimization procedure, the Jacobi parameters of the Jacobi expansion for random uncertainty analysis of acoustic systems with arbitrary probability distributions can be obtained.

4.2. Invert Response Law by Establishing Jacobian Extension. Random sound models can be fitted through the evolution of related parameters of variables, as follows:

$$
\begin{aligned}
& p_{k}(\xi)=\sum_{i_{1}=0}^{\infty} \ldots \sum_{i_{L}=0}^{\infty} f_{i_{1}, \ldots, i_{L}}^{k} J_{i_{1}, \ldots, i_{L}}^{(\alpha, \beta)}(\boldsymbol{\xi}), \\
& k=1,2, \ldots, N_{\text {tot }}
\end{aligned}
$$

where $p_{k}(\xi)\left(k=1,2, \ldots, N_{t o t}\right)$ denotes the sound pressure of acoustic field at the kth node, $N_{\text {tot }}$ is the number of nodes of the sound field, and $f_{i_{1}, \ldots, i_{L}}^{k}$ is the coefficient of Jacobi expansion to be estimated. In practice, the Jacobi expansion should be truncated at a finite order. Generally, the Jacobi expansion can be truncated by using total order expansion theory or the tensor order expansion theory. The tensor product expansion theory can effectively solve uncertain problems when the relevant retention order of 

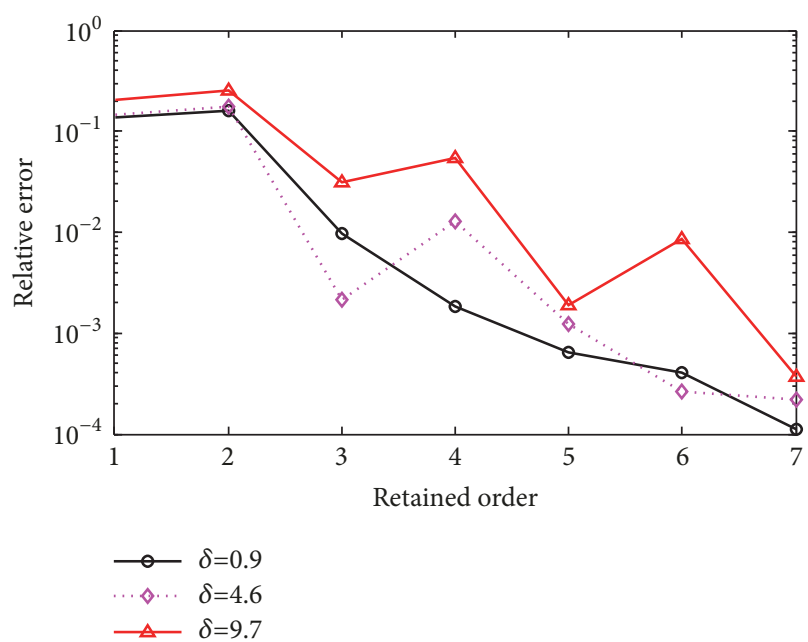

(a)

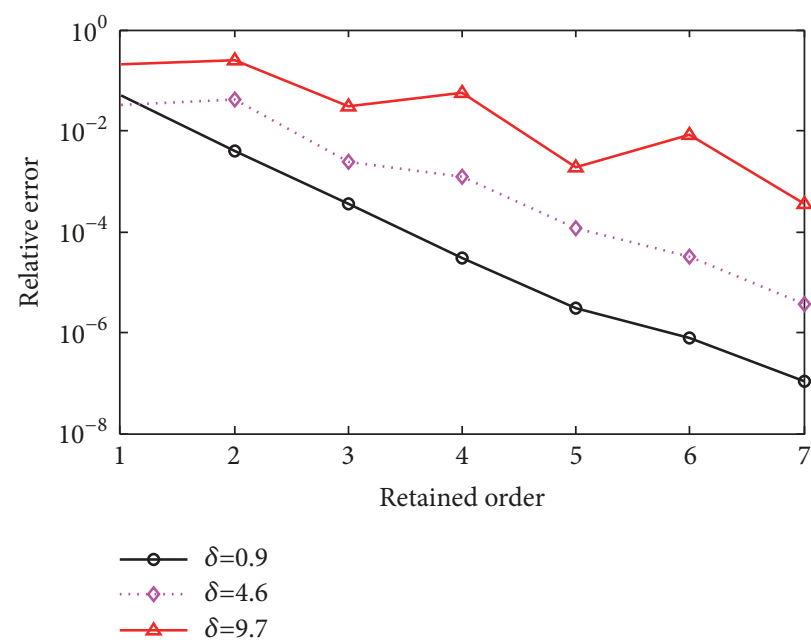

(b)

FIGURE 2: Convergence behavior of Jacobi expansion with different Jacobi parameters for the calculation of the mean and variance of y: (a) mean; (b) variance.

random variable changes [50]. The Jacobi expansion for the response of random acoustic model can be expressed as

$$
\begin{aligned}
p_{k}(\xi)=\sum_{i_{1}=0}^{N_{1}} \ldots \sum_{i_{L}=0}^{N_{L}} f_{i_{1}, \ldots, i_{L}}^{k} J_{i_{1}, \ldots, i_{L}}^{(\alpha, \beta)}(\boldsymbol{\xi}), & \\
& k=1,2, \ldots, N_{\text {tot }}
\end{aligned}
$$

where $N_{i}(i=1,2, \ldots, L)$ is the retained order of Jacobi expansion related to $\xi_{i}$.

For the type of tensor product expansion, the Gaussian integral method is very suitable to estimate the expansion characteristics [51]. Generally, the Gauss-Jacobi integration method is able to realize a reliable precision for the estimation of the expansion coefficient of Jacobi expansion When the Gauss-Jacobi integration point is equal to the expansion amount [35]. The coefficient $f_{i_{1}, \ldots, i_{L}}^{k}$ can be derived from the Gauss-Jacobi integral as

$$
\begin{gathered}
f_{i_{1}, \ldots, i_{L}}^{k} \approx \frac{1}{h_{1} \times \cdots \times h_{L}} \sum_{j_{1}=1}^{m_{1}} \cdots \sum_{j_{L}=1}^{m_{L}} p_{k}\left(\widehat{\xi}_{j_{1}}, \ldots, \widehat{\xi}_{j_{L}}\right) \\
\cdot J_{i_{1}, \ldots, i_{L}}^{(\alpha, \beta)}\left(\widehat{\xi}_{j_{1}}, \ldots, \widehat{\xi}_{j_{L}}\right) A_{j_{1}, \ldots, j_{L}}
\end{gathered}
$$

where $\left(\widehat{\xi}_{j_{1}}, \ldots, \widehat{\xi}_{j_{L}}\right)$ are the interpolating points and $\mathrm{m}_{\mathrm{i}}(\mathrm{i}=1,2, \ldots, \mathrm{L})$ is the amount of points of $\xi_{i}$. To reduce the deviation, the parameter $\mathrm{m}_{\mathrm{i}}$ is set as $\mathrm{N}_{\mathrm{i}}+1 . A_{j_{1}, j_{2}, \ldots, j_{L}}$ is the weighting value of Gauss-Jacobi integration, which can be expressed as

$$
A_{j_{1}, \ldots, j_{L}}=A_{j_{1}} \times A_{j_{2}} \times \cdots \times A_{j_{L}}
$$

In the above equation, the weight $A_{j_{i}}(i=1,2, \ldots, \mathrm{L})$ is given by [35]

$$
\begin{aligned}
A_{j_{i}}= & 2^{\alpha+\beta+1} \frac{\Gamma\left(m_{i}+\alpha+1\right) \Gamma\left(m_{i}+\beta+1\right)}{\Gamma\left(m_{i}+1\right) \Gamma\left(m_{i}+\alpha+\beta+1\right)}\left(1-x_{j}^{2}\right)^{-1} \\
& \cdot\left\{P_{m_{i}}^{(\alpha, \beta)^{\prime}}\left(x_{j}\right)\right\}^{-2}, \quad i=1,2, \ldots, m_{i}
\end{aligned}
$$

4.3. Estimation of Average and Variance. After the coefficient of Jacobi expansion is obtained, the average and variance of the response can be calculated approximately through the Jacobi method. The weight function of a Jacobi polynomial basis can be the same as the PDF of random variable. In this case, the IJCEM is identical to the traditional gPCEM. Thus, the mean and variance can be calculated as [30]

$$
\begin{aligned}
\mu_{p_{k}} & =f_{0,0, \ldots, 0}^{k} \\
\sigma_{p_{k}}^{2} & =\mathrm{E}\left[\left(p_{k}\right)^{2}\right]-\left(\mu_{p_{k}}\right)^{2} \\
& =\sum_{i_{1}=0}^{N_{1}} \cdots \sum_{i_{L}=0}^{N_{L}}\left(f_{i_{1}, \ldots, i_{L}}^{k}\right)^{2} h_{i_{1}, \ldots, i_{L}}-\left(f_{0,0, \ldots, 0}^{k}\right)^{2}
\end{aligned}
$$

For the random problems with nonbasic probability distribution, the mean and variance of response cannot be obtained analytically. To obtain the mean and variance of Jacobi expansion with uncertain variables, some numerical solvers can be utilized. In this paper, the mean and variance of the Jacobi expansion related to the random variable with nonbasic probability distribution are calculated through Monte Carlo simulation due to its simplicity. The additional computational cost suffering from MCM can be accepted in most practical engineering applications due to the simplification of the function.

Sometimes, both basic and nonbasic probability distributions may exist simultaneously. The response analysis 
of random problems with basic and nonbasic probability distributions includes two main steps. First, the random variable with nonbasic probability distribution is viewed as a fixed value, and the conditional mean and variance of Jacobi expansion with basic probability distributions can be obtained. For clarity, the Jacobi expansion with basic and nonbasic probability distributions can be expressed as

$$
\begin{array}{r}
p_{k}=\sum_{j_{1}=1}^{M_{1}} \ldots \sum_{j_{L_{2}}=1}^{M_{L_{2}}}\left(\sum_{i_{1}=0}^{N_{1}} \cdots \sum_{i_{L_{1}}=0}^{N_{L_{1}}} f_{i_{1}, \ldots, i_{L_{1}}, j_{1}, \ldots, j_{L_{2}}}^{k} G_{i_{1}, \ldots, i_{L_{1}}}(\boldsymbol{\eta})\right) \\
\cdot G_{j_{1}, \ldots, j_{L_{2}}}(\boldsymbol{\beta})=\sum_{j_{1}=1}^{M_{1}} \cdots \sum_{j_{L_{2}}=1}^{M_{L_{2}}} z_{i_{1}, \ldots, i_{L_{1}}, j_{1}, \ldots, j_{L_{2}}}^{k} G_{j_{1}, \ldots, j_{L_{2}}}(\boldsymbol{\beta})
\end{array}
$$

where

$$
z_{i_{1}, \ldots, i_{L_{1}}, j_{1}, \ldots, j_{L_{2}}}^{k}=\sum_{i_{1}=0}^{N_{1}} \ldots \sum_{i_{L}=0}^{N_{L_{1}}} f_{i_{1}, \ldots, i_{L_{1}}, j_{1}, \ldots, j_{L_{2}}}^{k} G_{i_{1}, \ldots, i_{L_{1}}}(\boldsymbol{\eta})
$$

In above equations, $\boldsymbol{\eta}$ and $\boldsymbol{\beta}$ denote the non-basic and basic random variable, respectively; $L_{1}$ is the number of $\boldsymbol{\eta}$ and $L_{2}$ for $\beta$.

Owing to the orthogonality of Jacobi polynomials, the mean and variance of $p_{k}$ related to the basic random variable $\beta$ are shown below

$$
\begin{aligned}
& \mu(\boldsymbol{\eta})=\sum_{i_{1}=0}^{N_{1}} \cdots \sum_{i_{L_{1}}=0}^{N_{L_{1}}} f_{i_{1}, \ldots, i_{L_{1}, 0 . \ldots, 0}}^{k} G_{i_{1}, \ldots, i_{L_{1}}}(\boldsymbol{\eta}) \\
& \sigma^{2}(\boldsymbol{\eta}) \\
& =\sum_{j_{1}=1}^{M_{1}} \cdots \sum_{j_{L_{2}}=1}^{M_{L_{2}}}\left(\sum_{i_{1}=1}^{N_{1}} \ldots \sum_{i_{L_{1}}=1}^{N_{L_{1}}} f_{i_{1}, \ldots, i_{L_{1}}, j_{1}, \ldots, j_{L_{2}}}^{k} G_{i_{1}, \ldots, i_{L_{1}}}(\boldsymbol{\eta})\right)^{2} \\
& \cdot h_{j_{1}, \ldots, j_{L_{2}}}-\left(\sum_{i_{1}=0}^{N_{1}} \ldots \sum_{i_{L_{1}}=0}^{N_{L_{1}}} f_{i_{1}, \ldots, i_{L_{1}, 0 . ., 0}}^{k} G_{i_{1}, \ldots, i_{L_{1}}}(\boldsymbol{\eta})\right)^{2}
\end{aligned}
$$

Then, the unconditional mean $\mu$ and the mean square value $\left(\mu^{2}+\sigma^{2}\right)$ of the response over the complete random ensemble can be evaluated using the laws of conditional probability

$$
\begin{aligned}
\mu & =\int \mu(\boldsymbol{\eta}) p(\boldsymbol{\eta}) d \boldsymbol{\eta}=\mathrm{E}[\mu(\boldsymbol{\eta})] \\
\mu^{2}+\sigma^{2} & =\int\left[\mu^{2}(\boldsymbol{\eta})+\sigma^{2}(\boldsymbol{\eta})\right] p(\boldsymbol{\eta}) d \boldsymbol{\eta} \\
& =\mathrm{E}\left[\mu^{2}(\boldsymbol{\eta})\right]+\mathrm{E}\left[\sigma^{2}(\boldsymbol{\eta})\right]
\end{aligned}
$$

where $\boldsymbol{\eta}$ are now considered to be random and described by a joint PDF $p(\boldsymbol{\eta})$. Equation (35) can be rewritten as

$$
\sigma^{2}=\mathrm{E}\left[\mu^{2}(\boldsymbol{\eta})\right]-\mathrm{E}^{2}[\mu(\boldsymbol{\eta})]+\mathrm{E}\left[\sigma^{2}(\boldsymbol{\eta})\right]
$$

By substituting $\sigma^{2}[\mu(\boldsymbol{\eta})]=\mathrm{E}\left[\mu^{2}(\boldsymbol{\eta})\right]-\mathrm{E}^{2}[\mu(\boldsymbol{\eta})]$ into (36), the unconditional variance is shown below

$$
\sigma^{2}=\sigma^{2}[\mu(\boldsymbol{\eta})]+\mathrm{E}\left[\sigma^{2}(\boldsymbol{\eta})\right]
$$

Subsequently, the mean and variance expressed in (34) and (37) can be derived with the Monte Carlo method. In particular, each item of (34) and (37) can be calculated by (32) or (33).

4.4. Procedure of IJCEM for Random Analysis of Sound with Arbitrary Probability Distributions. The procedures of IJCEM for the response analysis of acoustic systems with random parameters are shown below:

(1) Calculating the Jacobi parameter $\alpha$ and $\beta$ with respect to each random variable through the optimization solution shown in (22);

(2) The expansion coefficient is solved by (25)

(3) The integral weight is solved by (27)

(4) The solution of the response is given by (6)

(5) Calculate the mean and variance of response of sound field with different types of random variables. For the random variables with basic distributions, the mean and variance of responses are calculated through (28) and (29); for the random variables with nonbasic distributions, the mean and variance of responses are calculated through the Monte Carlo simulation; for the random variables with both basic and nonbasic distributions and the mean and variance of responses are calculated through (34) and (37).

\section{Numerical Examples}

\subsection{Acoustic Tube}

5.1.1. Random Model of an Acoustic Tube. As shown in Figure 3, a rigid two-dimensional sound tube finite element model is built and intermittent loads are applied to the inside of the model.

Considering the unpredictability of the environment temperature, $\rho, c$, and $v_{n}$ are considered as uncertain factors. For simplicity, these factors are considered as a function of fixed variables within a certain interval.

$$
\begin{aligned}
c & =\left(340+30 \xi_{1}\right) \mathrm{m} / \mathrm{s} \\
\rho & =\left[1.225+0.367\left(0.35 \xi_{2}+0.15 \xi_{2}^{3}\right)\right] \mathrm{kg} / \mathrm{m}^{3} \\
v_{n} & =\left[0.01+0.003\left(0.15 \xi_{3}+0.35 \xi_{3}^{5}\right)\right] \mathrm{m} / \mathrm{s}
\end{aligned}
$$

In the above equations, $\xi_{1}, \xi_{2}$, and $\xi_{3}$ are unitary random variables with Beta distribution. The equation for the Beta distribution is

$$
\begin{array}{r}
P^{(a, b)}(\xi)=\frac{\Gamma(a+b+1)}{2^{a+b+1} \Gamma(a+1) \Gamma(b+1)}(1+\xi)^{a}(1-\xi)^{b}, \\
-1 \leq \xi \leq 1
\end{array}
$$

where $\mathrm{a}$ and $\mathrm{b}$ are the Beta parameters, the Beta parameters of $\xi_{1}, \xi_{2}$, and $\xi_{3}$ are $a_{1}=b_{1}=4.5, a_{2}=b_{2}=3$, and $a_{3}=b_{3}=5$, respectively.

5.1.2. Comparison of the Accuracy of IJCEM and gPCEM under the Same Retained Order. According to the polynomial selection criterion of IJCEM, the Jacobi parameters of the 


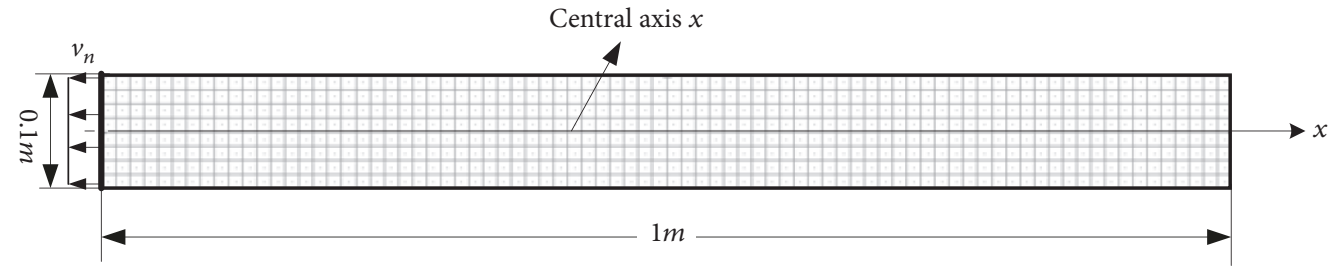

FIGURE 3: Mesh distribution of two-dimensional model.

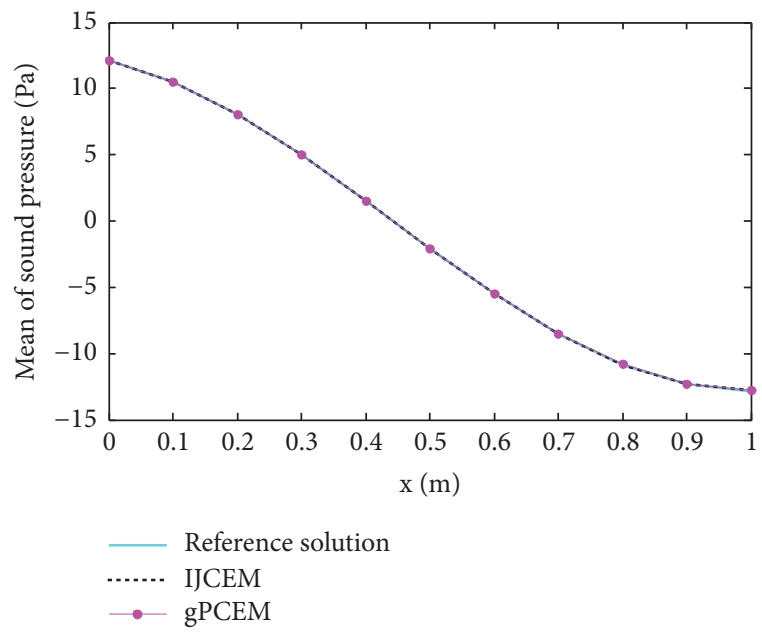

(a)

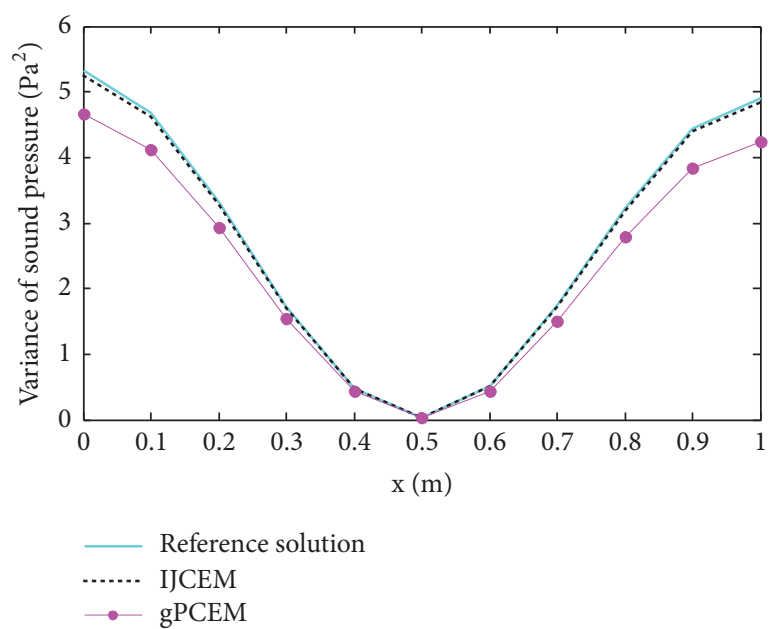

(b)

FIGURE 4: Mean and variance of the sound pressure's imaginary parts of acoustic tube along the central axis at $\mathrm{f}=150 \mathrm{~Hz}$ : (a) mean and (b) variance.

Jacobi expansion for $\rho, c$, and $v_{n}$ can be obtained, namely: $\alpha_{\rho}=\beta_{\rho}=4.5, \alpha_{c}=\beta_{c}=3.7$, and $\alpha_{v_{n}}=\beta_{v_{n}}=7.2 \mathrm{~A}$ relative improvement criterion will be introduced for random uncertainty analysis with IJCEM [51]. For multivariate random problems, the relative improvement of responses is given by [51]

$$
\operatorname{Ir}(\mathbf{k}, j)=\left|\frac{U\left(\mathbf{k}+\mathbf{e}^{j}\right)-U(\mathbf{k})}{U(\mathbf{k})}\right|
$$

where $\mathbf{k}=\left[k_{1}, k_{2}, \ldots, k_{L}\right]$ is the retained order vector, $k_{j}$ jth retention order, and $U(\mathbf{k})$ denotes the response calculated by using the Jacobi expansion with the $\mathbf{k}$ th retained orders. $\mathbf{e}_{j}$ is a L-dimensional vector in which only the jth element is equal to 1 , while the others are equal to zero. IJCEM is considered to be converged when $\operatorname{Ir}(\mathbf{k}, j)(\mathrm{j}=1,2, \ldots, \mathrm{L})$ is smaller than the customary prescribed tolerance. More detailed procedure of the promotion criteria for determining the retained order can be found in [51]. Assuming that the permissible relative error is $10^{-2}$, by using the promotion criteria, the order of IJCEM for random analysis of the model at $\mathrm{f}=150 \mathrm{~Hz}$ and $\mathrm{f}=300 \mathrm{~Hz}$ is

$$
\mathbf{N}_{r e q}=\left[N_{\rho}, N_{c}, N_{v_{n}}\right]=[1,2,1]
$$

The results of the sound pressure calculation with the retained order $[1,2,1]$ are shown in Figures 4 and 5, respectively. The reference solution is obtained by using the MCM, in which the number of sampling points is $10^{5}$. Note that the mean and variance of Jacobi expansion are also calculated by using the Monte Carlo simulation. In the Monte Carlo simulation of Jacobi expansion, the number of sampling points is also $10^{5}$. Besides, to show the accuracy of IJCEM and gPCEM more clearly, the relative errors of the mean and variance of responses integrated in different ways at $\mathrm{f}=150 \mathrm{~Hz}$ are listed in Table 2.

From Figures 4 and 5 and Table 2, we can find that the IJCEM is a high precision method. Besides, Table 2 has shown that the maximum value of relative error yielded by IJCEM is no more than $2 \%$, which retains high reliability. It illustrates the feasibility of applying the relative improvement criteria. By a comparison of the results obtained through the IJCEM and the gPCEM, it is proved that IJCEM is superior to gPCEM in the same condition. From Table 2, the relative error of the variance of responses yielded by gPCEM is more than $10 \%$. It indicates that higher order polynomial should be retained when the gPCEM is used to derive the variance of response of the model with random variables.

5.1.3. Comparison of the Efficiency of IJCEM and gPCEM under the Same Accuracy. For gPCEM, the required retained order can also be verified by using optimization guidelines. For random analysis of the acoustic tube at $\mathrm{f}=150 \mathrm{~Hz}$, the required retained order of gPCEM estimated is $[2,4,3]$. 


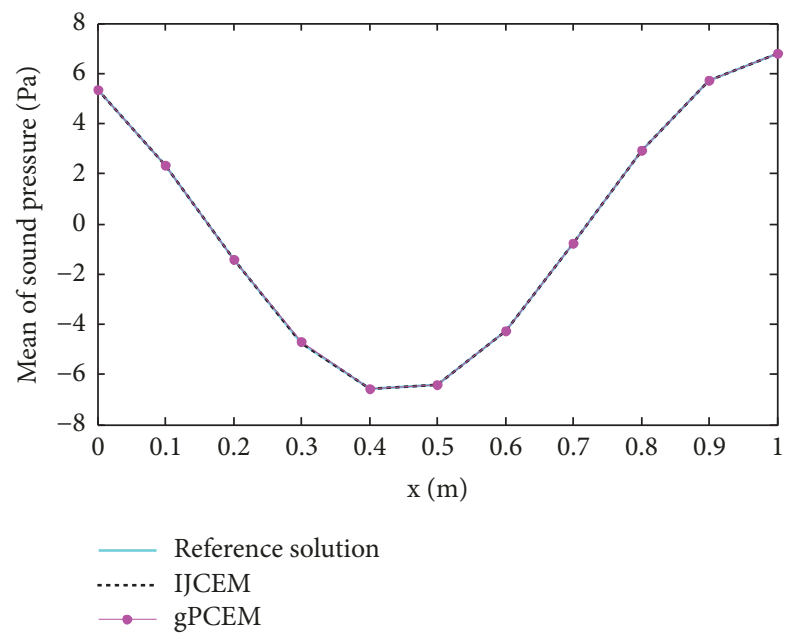

(a)

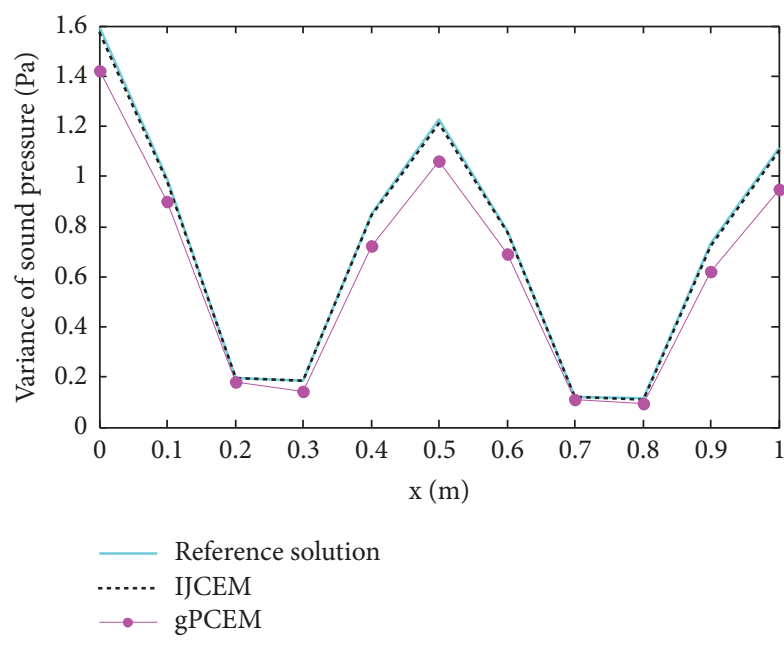

(b)

FIGURE 5: Mean and variance of the sound pressure's imaginary parts of acoustic tube along the central axis at $\mathrm{f}=300 \mathrm{~Hz}$ : (a) mean and (b) variance.

TABLE 2: Relative error of mean and variance of the sound pressure's imaginary parts.

\begin{tabular}{|c|c|c|c|c|}
\hline \multirow{2}{*}{$\mathrm{x}$} & \multicolumn{2}{|c|}{ Relative error of mean } & \multicolumn{2}{|c|}{ Relative error of variance } \\
\hline & IJCEM & gPCEM & IJCEM & gPCEM \\
\hline $0 \mathrm{~m}$ & $0.01 \%$ & $0.01 \%$ & $1.1 \%$ & $13.4 \%$ \\
\hline $0.2 \mathrm{~m}$ & $0.01 \%$ & $0.01 \%$ & $0.6 \%$ & $13.0 \%$ \\
\hline $0.5 \mathrm{~m}$ & $0.01 \%$ & $0.01 \%$ & $1.3 \%$ & $13.5 \%$ \\
\hline $1 \mathrm{~m}$ & $0.01 \%$ & $0.01 \%$ & $1.0 \%$ & $13.2 \%$ \\
\hline
\end{tabular}

TABLE 3: Relative error of variance of the sound pressure's imaginary parts yield by gPCEM.

\begin{tabular}{lccc}
\hline & MCM $(\mathrm{Pa})$ & gPCEM(Pa) & Relative error \\
\hline $0 \mathrm{~m}$ & 1.568 & 1.554 & $0.9 \%$ \\
$0.2 \mathrm{~m}$ & 0.195 & 0.193 & $1.0 \%$ \\
$0.5 \mathrm{~m}$ & 1.211 & 1.196 & $1.2 \%$ \\
$1 \mathrm{~m}$ & 1.102 & 1.087 & $1.4 \%$ \\
\hline
\end{tabular}

The variances of responses of the acoustic tube obtained by gPCEM are listed in Table 3.

From Table 3, we can find that the maximum value of relative errors yielded by gPCEM is $1.4 \%$. Thus, we can conclude that the criterion can be also used in gPCEM. Besides, the results shown in Table 3 indicate that the gPCEM can also achieve high accuracy for the response analysis of acoustic tube with random variables if the retained order is sufficiently large. However, the required retained order of gPCEM is higher than that of the IJCEM when the same accuracy is achieved.

The detailed execution time of gPCEM and IJCEM to calculate the response of acoustic tube at $f=150 \mathrm{~Hz}$ is listed in Table 4 . It can be found from Table 4 that the computational efficiency of IJCEM is greatly improved compared with the gPCEM. The main reason is that the required retained order of IJCEM is lower than that of the gPCEM, thus the corresponding computational time to construct the polynomial expansion can be decreased compared with the gPCEM. Besides, it can be seen from Table 4 that the computational time of Monte Carlo simulation of Jacobi expansion is much shorter than the total execution time of IJCEM. It indicates that the main computational burden of the proposed IJCEM for random problems suffers from the construction of the polynomial expansion. In other words, the additional time encountered from MCM of the Jacobi expansion is acceptable.

5.2. Acoustic Cavity of a Car. A finite element model was built, as shown in Figure 6. The acoustic cavity is surrounded by air with the density $\rho$ and the acoustic speed $c$. At the front windshield, the admittance coefficient along the Robin boundary is $A_{n}$. According to the characteristics of the front engine, the discontinuous normal velocity $v_{n}$ is imposed at the outside of the vocal cavity. The remaining edges are perfectly rigid. The finite element method is used to analyze the sound pressure of the acoustic cavity of the car. 
TABLE 4: Execution time comparison.

\begin{tabular}{lcccc}
\hline Methods & Retained order & $\begin{array}{c}\text { Execution time of the } \\
\text { construction of the } \\
\text { polynomial expansion }\end{array}$ & $\begin{array}{c}\text { Execution time of the Monte } \\
\text { Carlo simulation of the } \\
\text { polynomial expansion }\end{array}$ & Total execution time \\
\hline gPCEM & {$[2,4,3]$} & $60.2 \mathrm{~s}$ & $0 \mathrm{~s}$ & $60.5 \mathrm{~s}$ \\
IJCEM & {$[1,2,1]$} & $12.8 \mathrm{~s}$ & $2.5 \mathrm{~s}$ & $15.3 \mathrm{~s}$ \\
\hline
\end{tabular}

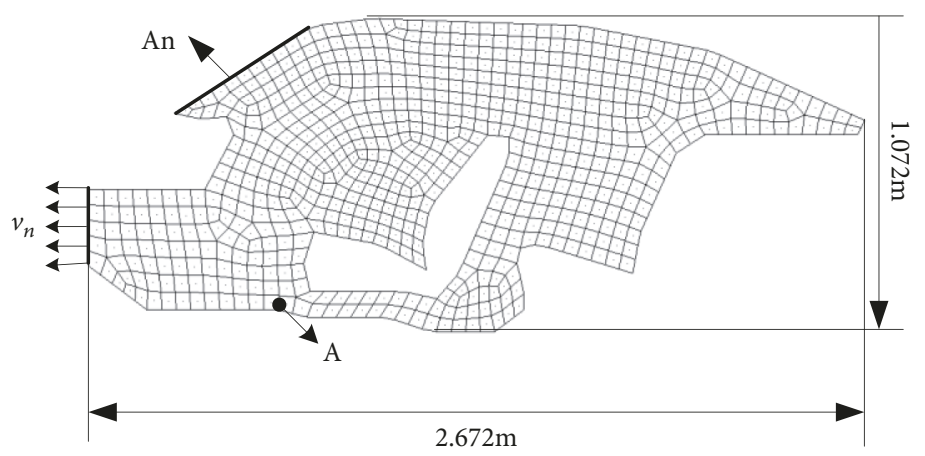

FIGURE 6: The 2D finite element mesh model of the acoustic cavity of a car.

The random parameters $\rho, c, A_{n}$, and $v_{n}$ are assumed as a function of the unitary random variable defined in $[-1,1]$ as follows:

$$
\begin{aligned}
& \text { Density of air: } \rho=1.225+0.367 \xi_{4} \\
& \text { Speed of sound: } c=340+20.5 \xi_{4} \\
& \text { Admittance coefficient: } A_{n}=0.0014+0.00042 \xi_{4} \\
& \text { Velocity of exciting: } v_{n}=0.01+0.003 \xi_{5}
\end{aligned}
$$

In order to compare the accuracy of the proposed method and gPCEM for the response analysis of random acoustic problems with complex PDFs, the PDF of $\xi_{4}$ and $\xi_{5}$ are assumed as a piecewise function. The original PDFs of $\xi_{4}$ and $\xi_{5}$ are shown in Figure 7 . For $\xi_{4}$ and $\xi_{5}$, the weight function from Askey scheme is difficult to approximate the original PDF. As a result, some errors may be introduced on the $\mathrm{PDF}$ of random variables by using gPCEM. For comparison, the PDF approximated by the weight function of polynomial basis in gPCEM is also plotted in Figure 7.

The proposed IJCEM and the gPCEM are employed to calculate the response of acoustic cavity of the car with random variables. The prescribed accuracy is set as $10^{-2}$. By using the relative improvement criterion, the required retained orders of IJCEM and gPCEM for random uncertainty analysis of the acoustic cavity of the car can be obtained as follows:

$$
\begin{gathered}
\mathbf{N}_{\text {req }}^{I J C E M}=\left[N_{\rho}, N_{c}, N_{A_{n}}, N_{v_{n}}\right]=[1,2,1,1], \\
\mathbf{N}_{\text {req }}^{g P C E M}=\left[N_{\rho}, N_{c}, N_{A_{n}}, N_{v_{n}}\right]=[1,3,1,1] .
\end{gathered}
$$

In the proposed IJCEM, the Jacobi parameters obtained according to the polynomial selection criterion are $\alpha_{1}=$ $\beta_{1}=6.2$ for $\rho, c$, and $A_{n}$ and $\alpha_{2}=\beta_{2}=5.5$ for $v_{n}$. The simulation results obtained by IJCEM and gPCEM are plotted in Figure 8 at $100 \mathrm{~Hz}$ and Figure 9 at $200 \mathrm{~Hz}$. The reference results obtained by using the MCM with $10^{5}$ sampling points are also plotted in Figures 8 and 9.

From Figures 8 and 9, we can find that the IJCEM can complete high precision calculation of acoustic cavity of car with random variables following piecewise PDFs. In contrast, the variance of response obtained by the gPCEM deviates significantly from the reference solution. The main reason is that the approximated PDF is used in gPCEM to calculate the mean and variance of the response of acoustic systems. Therefore, we can conclude that that the proposed IJCEM is more suitable for the random uncertainty analysis of acoustic problems than the gPCEM, especially when the PDF of random variables cannot be approximated by the weight function perfectly.

\section{Conclusion}

On the basis of the large family of Jacobi polynomials, the IJCEM is proposed to solve the random acoustic problem with arbitrary probability distributions. Jacobi expansion is used to fit the random problem. The coefficients of Jacobi expansion are calculated by the Gauss-Jacobi integration due to its robustness as well as its good efficiency for the estimation of the coefficients of tensor order polynomial expansion. By determining the effective polynomial selection criteria, the correlation weight function and the polynomial basis are obtained, and the response result can be effectively calculated. As the PDF of the random variable is not required to be expressed as the weight function of polynomial basis, the proposed IJCEM can avoid nonlinear transformation of variables for random problems with any complicated PDFs. Another advantage of the proposed IJCEM is that only the Jacobi parameters should be changed to construct the Jacobi expansion for the response analysis of acoustic systems with 


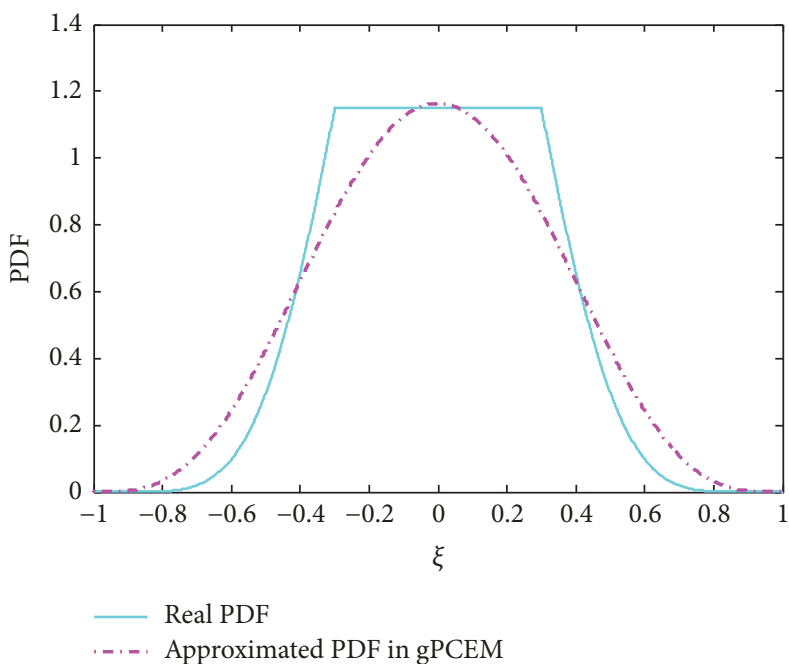

(a)

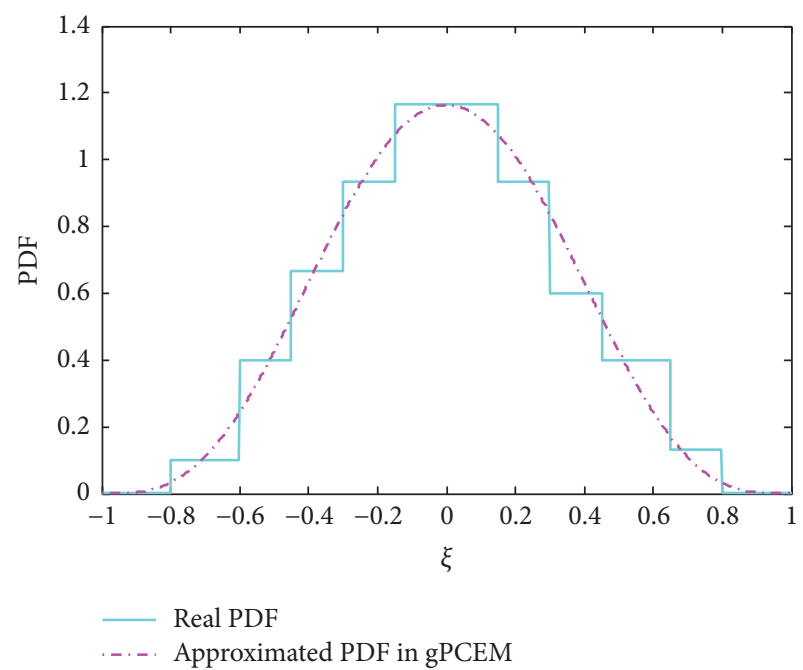

(b)

FIGURE 7: The original PDF of random variables and the approximated PDF in gPCEM: (a) $\xi_{4}$ and (b) $\xi_{5}$.

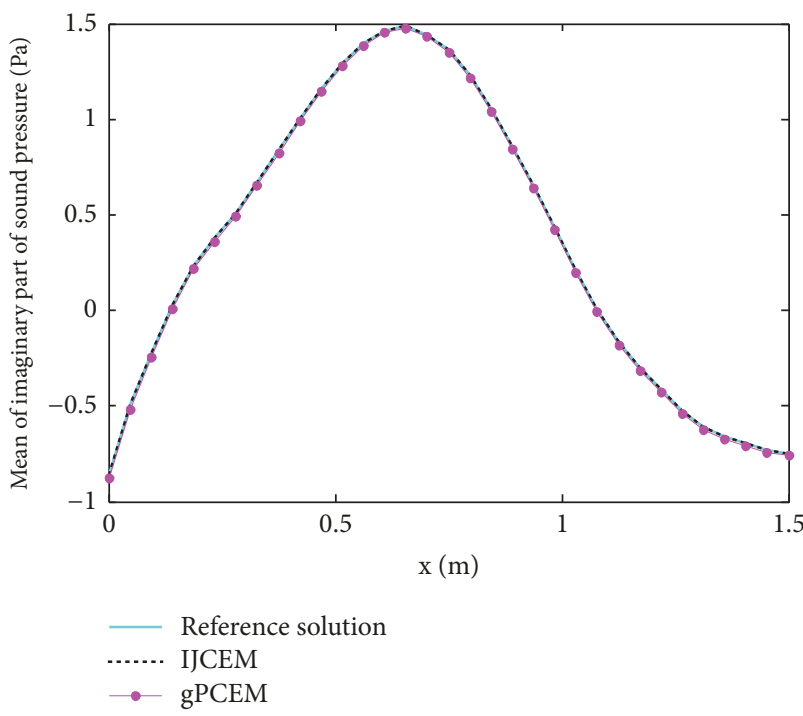

(a)

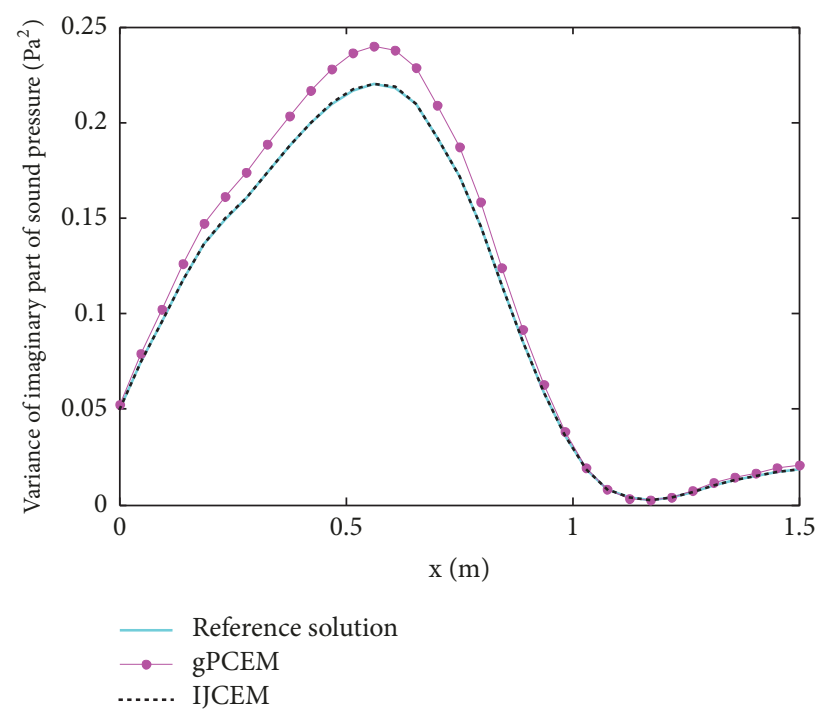

(b)

FIGURE 8: Mean and variance of sound pressure of car along the bottom boundary at $\mathrm{f}=100 \mathrm{~Hz}$ : (a) mean and (b) variance.

different random variables. Thus, the proposed method can be easily processed in the numerical implementation.

Two typical acoustic problems are used to investigate the effectiveness of the proposed methodology. The numerical results show that both IJCEM and gPCEM can achieve a desirable accuracy for the random uncertainty analysis of acoustic systems when the PDF of random variables can be represented in terms of the weight functions of polynomial basis. However, the computational efficiency of gPCEM is lower than that of the proposed IJCEM as the non-linear transformation is used for random variables in gPCEM. In addition, it can be concluded from the numerical study of random acoustic problems with complicated piecewise probability distributions that the accuracy of the gPCEM may deteriorate when some errors are introduced for PDFs by using the transformation of random variables. In contrast, the proposed IJCEM can achieve high accuracy for the random problem with arbitrary probability distributions if the retained order of Jacobi expansion is sufficiently large. Note that the mean and variance of Jacobi expansion are calculated by the Monte Carlo simulation when the weight function of polynomial basis is not identical to the PDF of random variables. However, compared with the improvement of the accuracy and efficiency in constructing the Jacobi expansion, the additional computational cost suffering from the Monte Carlo simulation of Jacobi expansion is acceptable, especially for large scale engineering problems. It should be also pointed out that the proposed IJCEM is not limited to the 


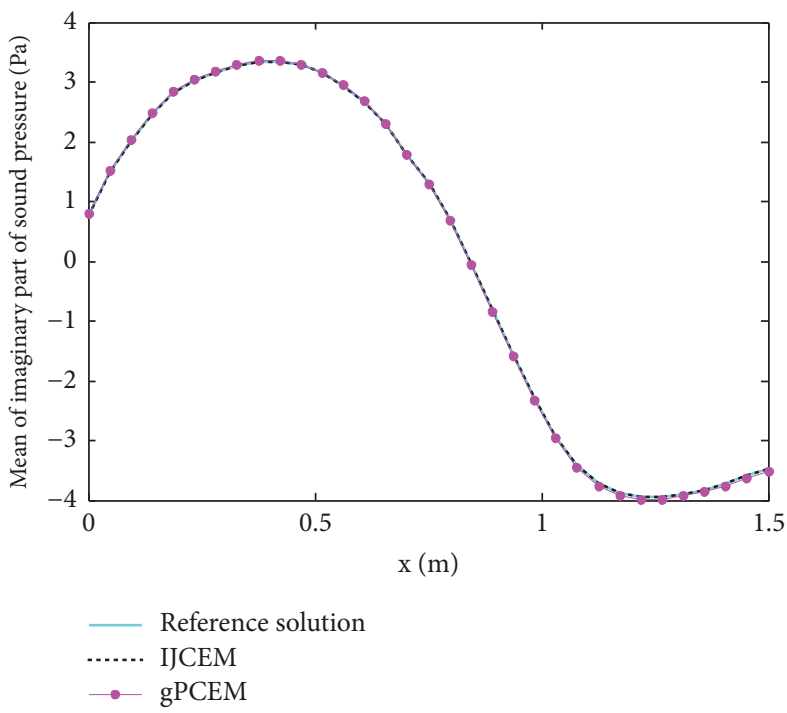

(a)

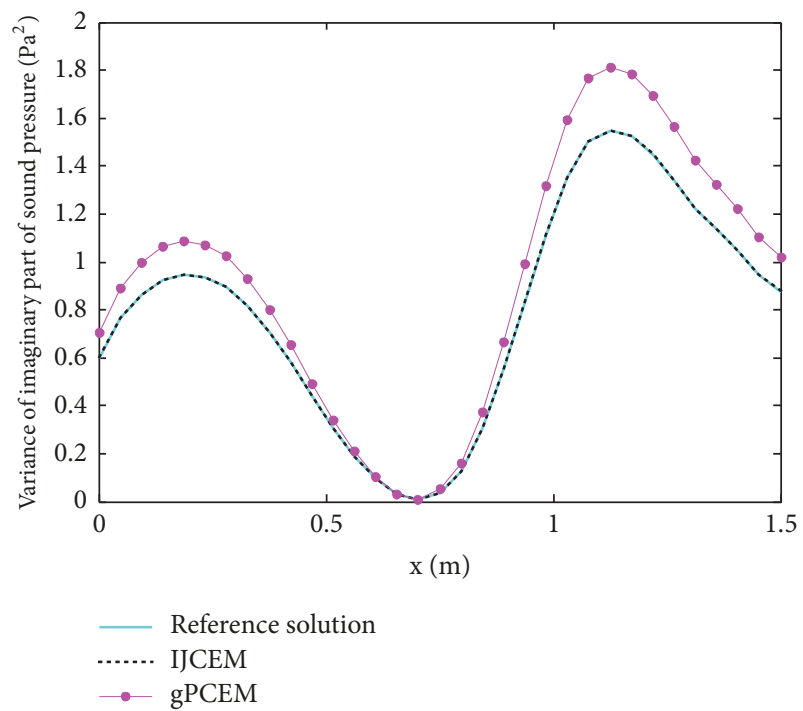

(b)

FIgURE 9: Mean and variance of sound pressure of car along the bottom boundary at $f=200 \mathrm{~Hz}$ : (a) mean and (b) variance.

response analysis of acoustic systems. With a suitable extension, it can be used for response analysis in multidisciplinary computational mechanics.

It is noted that the computational cost of IJCEM will increase rapidly with the number of random variables. Thus, the IJCEM is not suitable for random problem with large number of random variables.

\section{Data Availability}

The data used by the researchers are for scientific research purposes rather than commercial purposes. Most of the data generated or analysed during this study are included in this manuscript and all of the data are available from the corresponding author on reasonable request.

\section{Conflicts of Interest}

The authors of this study state that there are no conflicts of interest to disclose.

\section{Acknowledgments}

This work was supported by the National Natural Science Foundation of China (51774323).

\section{References}

[1] B. Xia, S. Yin, and D. Yu, "A new random interval method for response analysis of structural-acoustic system with interval random variables," Applied Acoustics, vol. 99, pp. 31-42, 2015.

[2] B. Xia and D. Yu, "Optimization based on reliability and confidence interval design for the structural-acoustic system with interval probabilistic variables," Journal of Sound and Vibration, vol. 336, pp. 1-15, 2015.
[3] B. Xia, H. Yin, and D. Yu, "Nondeterministic wave-based methods for low- and mid-frequency response analysis of acoustic field with limited information," Mechanical Systems and Signal Processing, vol. 84, pp. 169-183, 2017.

[4] I. Harari, "A survey of finite element methods for timeharmonic acoustics," Computer Methods Applied Mechanics and Engineering, vol. 195, no. 13-16, pp. 1594-1607, 2006.

[5] K. R. James and D. R. Dowling, "A method for approximating acoustic-field-amplitude uncertainty caused by environmental uncertainties," The Journal of the Acoustical Society of America, vol. 124, no. 3, pp. 1465-1476, 2008.

[6] G. Stefanou, "The stochastic finite element method: past, present and future," Computer Methods Applied Mechanics and Engineering, vol. 198, no. 9-12, pp. 1031-1051, 2009.

[7] J. Novick and S. Finette, "Stochastic basis expansions applied to acoustic propagation in an uncertain, range, and depth-dependent, multi-layered waveguide.," The Journal of the Acoustical Society of America, vol. 129, no. 4, pp. 2600-2600, 2011.

[8] M. Beer, S. Ferson, and V. Kreinovich, "Imprecise probabilities in engineering analyses," Mechanical Systems and Signal Processing, vol. 37, no. 1-2, pp. 4-29, 2013.

[9] D. Moens and D. Vandepitte, "A survey of non-probabilistic uncertainty treatment in finite element analysis," Computer Methods Applied Mechanics and Engineering, vol. 194, no. 12-16, pp. 1527-1555, 2005.

[10] A. Sofi and E. Romeo, "A novel interval finite element method based on the improved interval analysis," Computer Methods Applied Mechanics and Engineering, vol. 311, pp. 671-697, 2016.

[11] G. Shafer, A Mathematical Theory of Evidence, Princeton University Press, Princeton, NJ, USA, 1976.

[12] Y. Y. Khine, D. B. Creamer, and S. Finette, "Acoustic propagation in an uncertain waveguide environment using stochastic basis expansions," Journal of Computational Acoustics, vol. 18, no. 4, pp. 397-441, 2010.

[13] J. E. Hurtado and D. A. Alvarez, “The encounter of interval and probabilistic approaches to structural reliability at the design 
point," Computer Methods Applied Mechanics and Engineering, vol. 225-228, pp. 74-94, 2012.

[14] A. Secgin, J. F. Dunne, and L. Zoghaib, "Extreme-ValueBased Statistical Bounding of Low, Mid, and High Frequency Responses of a Forced Plate With Random Boundary Conditions," Journal of Vibration and Acoustics, vol. 134, no. 2, p. 021003, 2012.

[15] "Monte Carlo and Quasi-Monte Carlo Methods in Scientific Computing," Technometrics, vol. 38, no. 4, pp. 414-414, 1996.

[16] J. C. HELTON and F. J. DAVIS, "Latin Hypercube Sampling and the Propagation of Uncertainty in Analyses of Complex Systems," Tech. Rep. SAND2001-0417, 2002.

[17] G. I. Schuëller, H. J. Pradlwarter, and P. S. Koutsourelakis, "A critical appraisal of reliability estimation procedures for high dimensions," Probabilistic Engineering Mechanics, vol. 19, no. 4, pp. 463-474, 2004.

[18] S. K. Au and J. L. Beck, "Subset simulation and its application to seismic risk based on dynamic analysis," Journal of Engineering Mechanics, vol. 129, no. 8, pp. 901-917, 2003.

[19] P. S. Koutsourelakis, H. J. Pradlwarter, and G. I. Schuëller, "Reliability of structures in high dimensions, part I: Algorithms and applications," Probabilistic Engineering Mechanics, vol. 19, no. 4, pp. 409-417, 2004.

[20] D. Xiu, "Fast numerical methods for stochastic computations: a review," Communications in Computational Physics, vol. 5, no. 2-4, pp. 242-272, 2009.

[21] M. N. Ichchou, F. Bouchoucha, M. A. Ben Souf, O. Dessombz, and M. Haddar, "Stochastic wave finite element for random periodic media through first-order perturbation," Computer Methods Applied Mechanics and Engineering, vol. 200, no. 41-44, pp. 2805-2813, 2011.

[22] M. Kaminski, "Stochastic second-order perturbation approach to the stress-based finite element method," International Journal of Solids and Structures, vol. 38, no. 21, pp. 3831-3852, 2001.

[23] X. G. Hua, Y. Q. Ni, Z. Q. Chen, and J. M. Ko, "An improved perturbation method for stochastic finite element model updating," International Journal for Numerical Methods in Engineering, vol. 73, no. 13, pp. 1845-1864, 2008.

[24] A. Culla and A. Carcaterra, "Statistical moments predictions for a moored floating body oscillating in random waves," Journal of Sound and Vibration, vol. 308, no. 1-2, pp. 44-66, 2007.

[25] W. Gao, N. Zhang, and J. Ji, "A new method for random vibration analysis of stochastic truss structures," Finite Elements in Analysis and Design, vol. 45, no. 3, pp. 190-199, 2009.

[26] M. Kamiński, "Probabilistic entropy in homogenization of the periodic fiber-reinforced composites with random elastic parameters," International Journal for Numerical Methods in Engineering, vol. 90, no. 8, pp. 939-954, 2012.

[27] D. Xiu and G. E. Karniadakis, "Modeling uncertainty in flow simulations via generalized polynomial chaos," Journal of Computational Physics, vol. 187, no. 1, pp. 137-167, 2003.

[28] R. Ghanem, "Scales of fluctuation and the propagation of uncertainty in random porous media," Water Resources Research, vol. 34, no. 9, pp. 2123-2136, 1998.

[29] R. Ghanem, "Ingredients for a general purpose stochastic finite elements implementation," Computer Methods Applied Mechanics and Engineering, vol. 168, no. 1-4, pp. 19-34, 1999.

[30] D. Xiu and G. E. Karniadakis, "Modeling uncertainty in steady state diffusion problems via generalized polynomial chaos," Computer Methods Applied Mechanics and Engineering, vol. 191, no. 43, pp. 4927-4948, 2002.
[31] D. Xiu and G. E. Karniadakis, “The wiener-askey polynomial chaos for stochastic differential equations," SIAM Journal on Scientific Computing, vol. 24, no. 2, pp. 619-644, 2002.

[32] D. Xiu and G. E. Karniadakis, "A new stochastic approach to transient heat conduction modeling with uncertainty," International Journal of Heat and Mass Transfer, vol. 46, no. 24, pp. 4681-4693, 2003.

[33] Y. A. Brychkov, Handbook of special functions: derivatives, integrals, series and other formulas, CRC Press, New York, NY, USA, 2008.

[34] N. Wiener, "The homogeneous chaos," American Journal of Mathematics, vol. 60, no. 4, pp. 897-936, 1938.

[35] G. Szegö, Orthogonal Polynomials, American Mathematical Society, 4th edition, 1975.

[36] N. Li, J. Zhao, X. Feng, and D. Gui, "Generalized polynomial chaos for the convection diffusion equation with uncertainty," International Journal of Heat and Mass Transfer, vol. 97, pp. 289300, 2016.

[37] B. Sudret and C. Mai, "Computing derivative-based global sensitivity measures using polynomial chaos expansions," Reliability Engineering \& System Safety, vol. 134, pp. 241-250, 2015.

[38] P. L. Duong, W. Ali, E. Kwok, and M. Lee, "Uncertainty quantification and global sensitivity analysis of complex chemical process using a generalized polynomial chaos approach," Computers \& Chemical Engineering, vol. 90, pp. 23-30, 2016.

[39] X. Wan and G. E. Karniadakis, "Error control in multi-element generalized polynomial chaos method for elliptic problems with random coefficients," Communications in Computational Physics, vol. 5, no. 2-4, pp. 793-820, 2009.

[40] X. Wan and G. E. Karniadakis, "Multi-element generalized polynomial chaos for arbitrary probability measures," SIAM Journal on Scientific Computing, vol. 28, no. 3, pp. 901-928, 2006.

[41] C. Wang, H. G. Matthies, M. Xu, and Y. Li, "Dual interval-andfuzzy analysis method for temperature prediction with hybrid epistemic uncertainties via polynomial chaos expansion," Computer Methods Applied Mechanics and Engineering, vol. 336, pp. 171-186, 2018.

[42] C. Wang, Z. Qiu, and Y. Yang, "Uncertainty propagation of heat conduction problem with multiple random inputs," International Journal of Heat and Mass Transfer, vol. 99, pp. 95$101,2016$.

[43] C. Wang, Z. Qiu, and Y. Yang, "Collocation methods for uncertain heat convection-diffusion problem with interval input parameters," International Journal of Thermal Sciences, vol. 107, pp. 230-236, 2016.

[44] R. P. Dwight, J. A. Witteveen, and H. Bijl, "Adaptive Uncertainty Quantification for Computational Fluid Dynamics," in Uncertainty Quantification in Computational Fluid Dynamics, vol. 92 of Lecture Notes in Computational Science and Engineering, pp. 151-191, Springer International Publishing, Cham, 2013.

[45] S. Yin, D. Yu, H. Yin, and B. Xia, "A unified method for the response analysis of interval/random variable models of acoustic fields with uncertain-but-bounded parameters," International Journal for Numerical Methods in Engineering, vol. 111, no. 6, pp. 503-528, 2017.

[46] C. Wang and Z. Qiu, "An interval perturbation method for exterior acoustic field prediction with uncertain-but-bounded parameters," Journal of Fluids and Structures, vol. 49, pp. 441449, 2014. 
[47] B. Xia, D. Yu, and J. Liu, "Hybrid uncertain analysis for structural-acoustic problem with random and interval parameters," Journal of Sound and Vibration, vol. 332, no. 11, pp. 27012720, 2013.

[48] B. Xia and D. Yu, "Response probability analysis of random acoustic field based on perturbation stochastic method and change-of-variable technique," Journal of Vibration and Acoustics, vol. 135, no. 5, Article ID 051032, 2013.

[49] B. Xia, D. Yu, and J. Liu, "Probabilistic interval perturbation methods for hybrid uncertain acoustic field prediction," Journal of Vibration and Acoustics, vol. 135, no. 2, Article ID 021009, 2013.

[50] M. S. Eldred, "Recent advances in non-intrusive polynomial chaos and stochastic collocation methods for uncertainty analysis and design," in Proceedings of the 50th AIAA/ASME/ASCE/AHS/ASC Structures, Structural Dynamics, and Materials Conference, AIAA Paper 2009-2274, Palm Springs, Calif, USA, 2009.

[51] S. Yin, D. Yu, H. Yin, and B. Xia, "Interval and random analysis for structure-acoustic systems with large uncertain-butbounded parameters," Computer Methods Applied Mechanics and Engineering, vol. 305, pp. 910-935, 2016. 


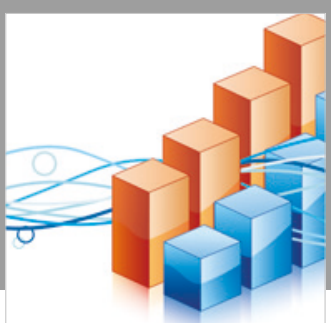

Advances in

Operations Research

\section{-n-m}
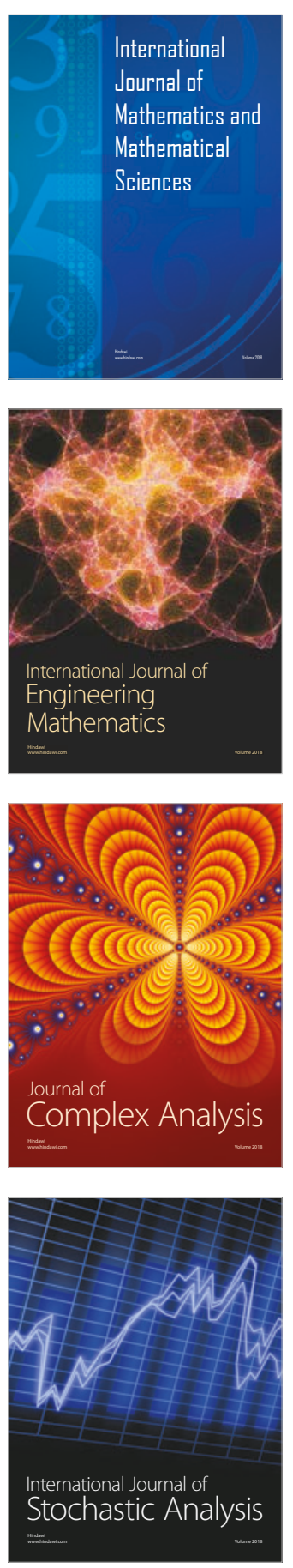
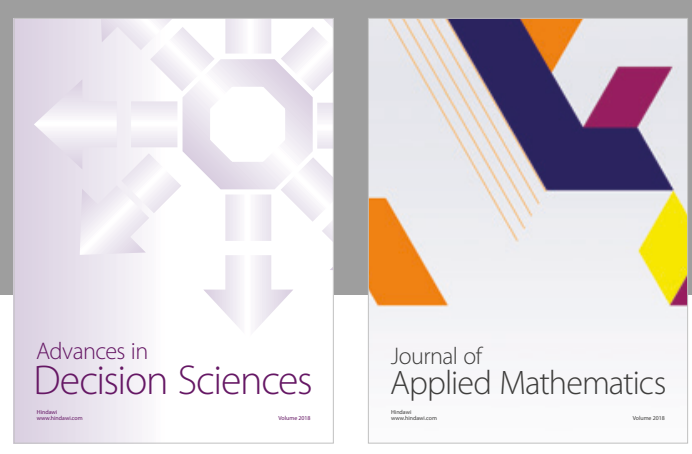

Journal of

Applied Mathematics
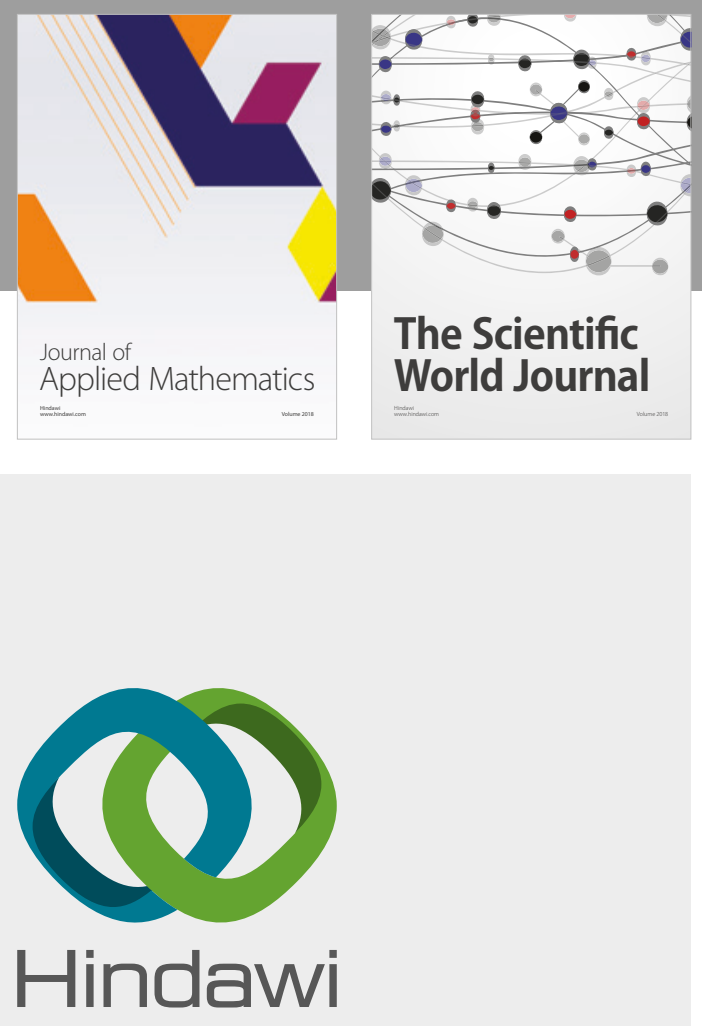

Submit your manuscripts at

www.hindawi.com

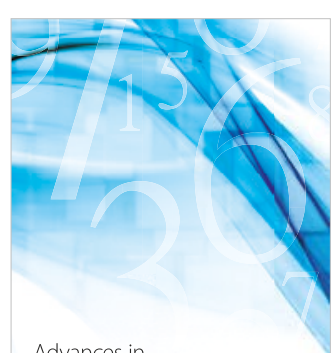

Advances in
Numerical Analysis
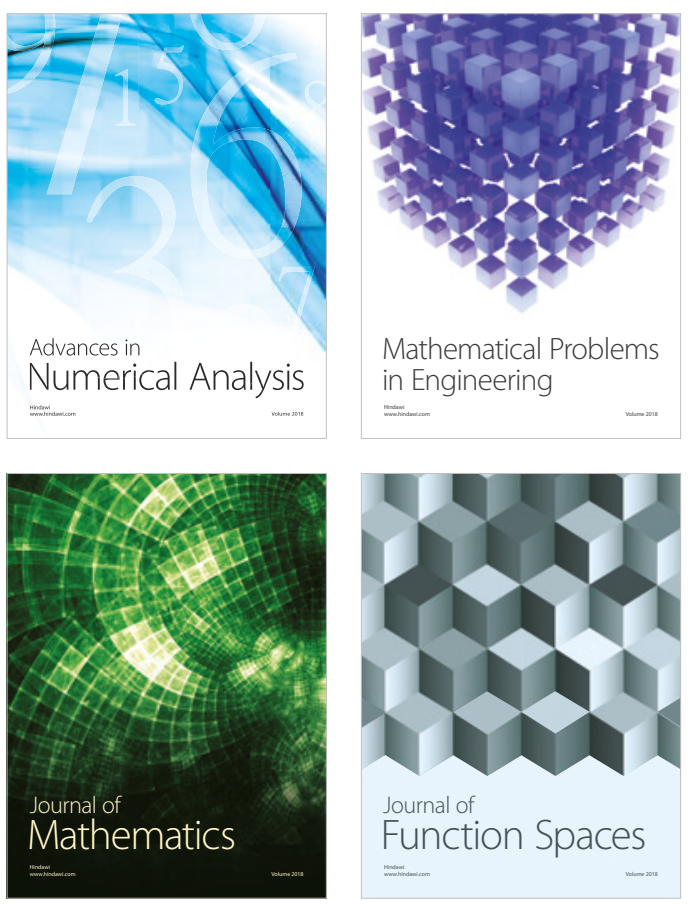

Mathematical Problems in Engineering

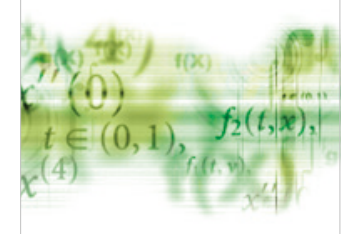

International Journal of

Differential Equations

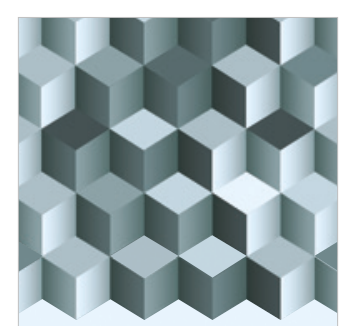

Journal of

Function Spaces
The Scientific

World Journal

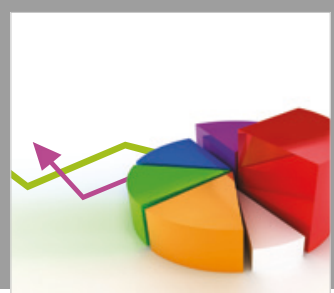

Journal of

Probability and Statistics
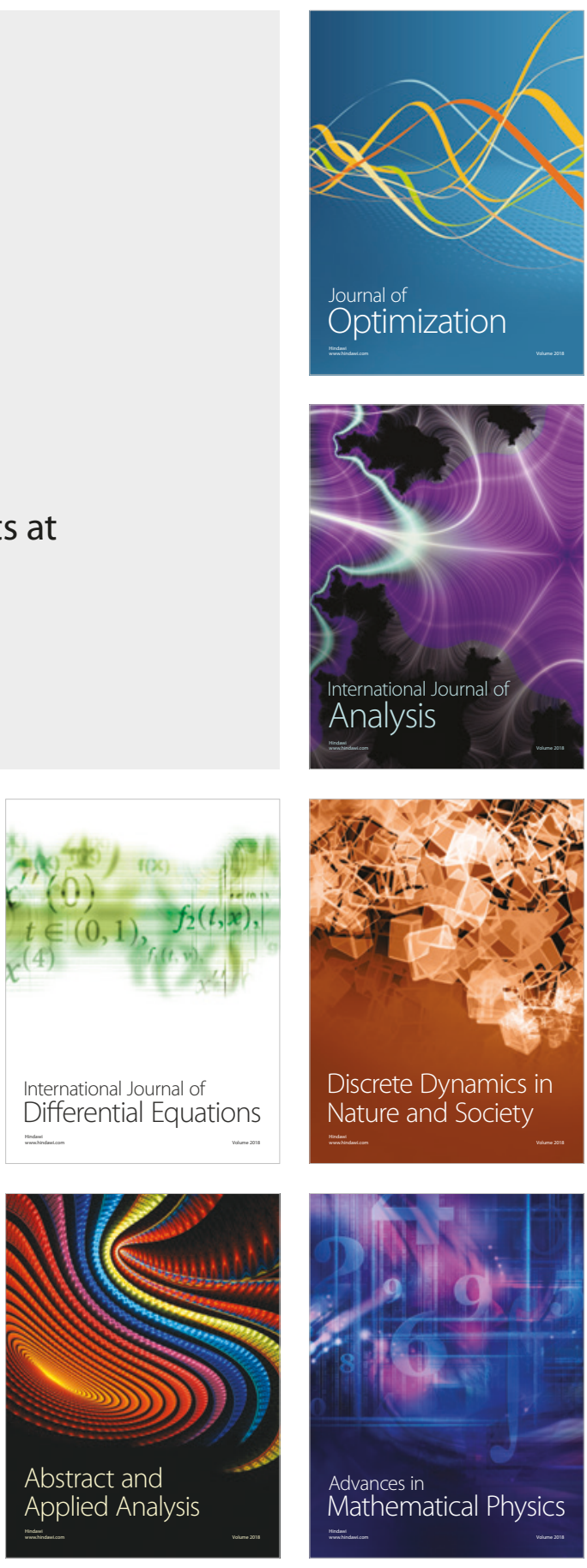